\title{
The Calculation of Inductor Sizes, for Heat Treatment of Aluminum Wires
}

\author{
Ayman Y. Al-Rawashdeh \\ Al-Balqa`a Applied University
}

\begin{abstract}
Aluminum is efficient in using as a conducting material when producing wires and cables because of its high electrical conductivity and low specific gravity. Wires of different diameters are used for the production of the conductors, drawn from aluminum rods. Heat treatment of the wires is necessary for recovering the ductility of the conductors' material. The progressive way of increasing the aluminum wires ductility is prolonged induction of heat treatment, which is based upon using an inductor with a ferromagnetic core, with one of its rods covering the circuit, drawn from the portion of the heated wire, and short-circuiting on the deflecting rollers. Calculations of basic dimensions, choosing the core material, and the inductors' winding is determined by its weight, size and cost indicators. In this paper the initial data for calculations are; the performance of the heat treatment process, the power supply voltage and temperature, at which the ductile properties of aluminum are recovered.
\end{abstract}

Keyword: Simulink/Matlab, Heat Treatment, Conductor, Cross Sectional Dimension.

\section{Introduction}

Many factors determine the type of electrical conductor used to connect components. Some of these factors are the physical size of the conductor, its composition, and its electrical characteristics. Other factors that can determine the choice of a conductor are the weight, the cost, and the environment where the conductor will be used. To compare the resistance and size of one conductor with that of another, we need to establish a standard or unit size. A convenient unit of measurement of the diameter of a conductor is the mil $(0.001$, or one-thousandth of an inch). A convenient unit of conductor length is the foot. The standard unit of size in most cases is the MIL-FOOT. A wire will have a unit size if it has a diameter of 1 mil and a length of 1 foot.

Specific resistance, or resistivity, is the resistance in ohms offered by a unit volume (the circular-mil- foot or the centimeter cube) of a substance to the flow of electric current. Resistivity is the reciprocal of conductivity. A substance that has a high resistivity will have a low conductivity, and vice versa. Thus, the specific resistance of a substance is the resistance of a unit volume of that substance.

Many tables of specific resistance are based on the resistance in ohms of a volume of a substance 1 foot in length and 1 circular mil in cross-sectional area. The temperature at which the resistance measurement is made is also specified. If you know the kind of metal a conductor is made of, you can obtain the specific resistance of the metal from a table. The specific resistances of some common substances are given in table 1-1.

Table 1-1.-Specific Resistances of Common Substances

\begin{tabular}{|l|c|c|}
\hline \multirow{2}{*}{ Substance } & \multicolumn{2}{|c|}{ Specific resistance at $20^{\circ} \mathrm{C}}$. \\
\cline { 2 - 3 } & $\begin{array}{c}\text { Centimeter cube } \\
\text { (microhoms) }\end{array}$ & $\begin{array}{c}\text { Circular-mil-foot } \\
\text { (ohms) }\end{array}$ \\
\hline Silver & 1.629 & 9.8 \\
\hline Copper (drawn) & 1.724 & 10.37 \\
\hline Gold & 2.44 & 14.7 \\
\hline Aluminum & 2.828 & 17.02 \\
\hline Carbon (amorphous) & 3.8 to 4.1 & $\ldots \ldots \ldots \ldots \ldots \ldots \ldots$ \\
\hline Tungsten & 5.51 & 33.2 \\
\hline Brass & 7.0 & 42.1 \\
\hline Steel (soft) & 15.9 & 95.8 \\
\hline Nichrome & 109.0 & 660.0 \\
\hline
\end{tabular}


The resistance of a conductor of a uniform cross section varies directly as the product of the length and the specific resistance of the conductor, and inversely as the cross-sectional area of the conductor. Therefore, you can calculate the resistance of a conductor if you know the length, cross-sectional area, and specific resistance of the substance. See fig. 1.

Many papers discuss the specifications of aluminum wires, Robert L. D. et al. 2010, performed armor simulations with quantifiable confidence, to validate material models by comparing a variety of computational data with carefully measured experiments. Exploding wires serve as excellent tests of material models since one can tune the energy deposition so that the wire passes through solid, liquid, and gas phases. The experimental data collected includes electrical measurements where material properties vary over a wide range of temperature, pressures, and electrical conductivities. Tthis work focused on aluminum wires immersed in a water environment. The simulations showed outstanding agreement with experiment when one factors in measurement uncertainty. It is found great sensitivity with certain parameters, which lead to large variability in the results at late time.
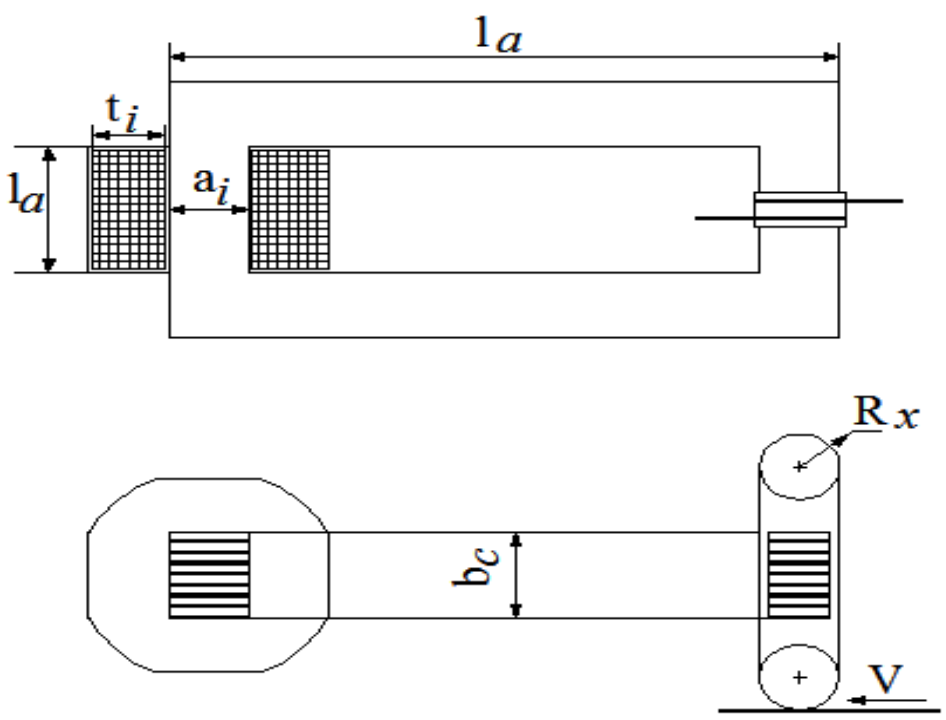

Fig.1. The inductor for heat treatment of aluminum wires.

Performance of the process is completely determined by the speed of the wire feed, the choice of power supply is linked to the choice of frequency. The main dimensions of the inductor (picture.1) are connected through the following relations:

The length of the heated portion of the wire

$$
l_{k}=2 \pi \cdot R_{k}+4\left(R_{k}+\sigma\right)+2 b_{c}
$$

Where

$R_{k}$ - the radius of the deflecting rollers,

$b_{c}$ - the thickness of the core package,

$\sigma$ - size of the gap between the surface of the core and the rim of the roller.

The length of the average coil winding

$l_{c p}=2\left(a_{c}+b_{c}\right)+\pi b_{0}$

Where

$$
\begin{aligned}
& a_{c}-\text { the width of the plate core; } \\
& b_{0}-\text { the width of the cross-section of the winding. }
\end{aligned}
$$

the value of $l_{c p}$ determines the consumption of copper in the windings of the inductor and depends on the ratio of the cores' cross sections, in addition to that the size of the cross section is determined by the cross sectional area of the inductors' rod core 
$s_{c}=a_{c} \cdot b_{c}$

At a given value $S_{c}$ the length of an average loop

$l_{c p}=2\left(a_{c}+\frac{s_{c}}{a_{c}}\right)+\pi b_{0}$

Terms of the vanishing of the derivative $\frac{d l_{c r}}{d a_{c}}$, taken from equation (4), is the condition for the minimum $l_{c p}$ with the given value of the sectional area of the core

$$
l_{c p} \frac{d l_{c r}}{d a_{c}}=1-\frac{s_{c}}{a_{c}^{2}}=0
$$

Whence, $a_{c}^{2}=S_{\sigma}$, consequently, the sectional shape of the rods' core - is a square, and besides

that $a_{c}=b_{c}$.

Since $R_{K}$ is much bigger than $\sigma$, it's permitted to assume that

$l_{k}=(2 \pi+6) \cdot R_{K}$

It is necessary to provide a protective interval $\sigma_{1}$, between the surfaces of the core and wire, therefore,

$$
a_{c}=2\left(R_{k}-\delta 1\right)=2 R_{k}\left(1-\frac{\delta_{1}}{R_{k}}\right)=2 \cdot k_{1} \cdot R_{k}
$$

$\sigma_{1}$ value is selected, so that during movement the wire doesn't touch the surface of the rod on the area of the circuit between the deflection rollers (picture.1), for that usually $R_{k}=10 \mathrm{~mm}$ is enough.

Now expression (2) can be formed this way

$$
l_{c p}=8 k_{1} \cdot R_{k}+\pi b_{0}
$$

The area of the cross section of the rod's core is also expressed as function $R_{k}$

$$
s_{c}=4 k_{1}^{2} \cdot R_{k}^{2}
$$

The balance of the heating circuit's power when neglecting heat loss takes the form of

$$
\frac{E_{k}^{2}}{R_{3}}=c \cdot \gamma \cdot q \cdot v\left(\theta_{2}-\theta_{1}\right)
$$

Where

$E_{k}$ - electromotive force of the circuit;

$R_{k}$ - the resistance of the circuit;

$c$ - the specific heat of aluminum;

$\gamma$ - the density of aluminum;

$q$ - the cross sectional area of the wire;

$v$ - wire feed speed;

$\theta_{2}$ - the temperature of the wire at the outlet of the inductor;

$\theta_{1}$ - the temperature of the wire at the inlet of the inductor.

as shown in (2), the value of the active resistance of the circuit is given by 


$$
R_{3}=\rho_{A} \cdot \frac{\alpha\left(\theta_{2}-\theta_{1}\right)}{\ln \frac{1+\alpha \theta_{2}}{1+\alpha \theta_{1}}} \cdot \frac{l_{k}}{q}
$$

Where

$\rho_{A}-$ the resistivity of aluminum;

$\alpha$ - the temperature coefficient of the aluminums' resistance;

$\theta_{2}-$ the temperature of the wire at the outlet of the inductor;

$\theta_{1}$ - the temperature of the wire at the inlet of the inductor;

$l_{k}-$ the length of the circuit.

if we assume that $\theta_{2}=250^{\circ} \mathrm{C}$, and $\theta_{1}=15^{\circ} \mathrm{C}$, then

$$
R_{3}=1,4 \cdot \rho_{A} \frac{l_{k}}{q}
$$

The effective value of the electromotive force, which is present in the expression (10), can also be expressed in the following form

$$
E_{k}=\frac{\omega \cdot \Phi_{m}}{2^{\frac{1}{2}}}=\frac{\omega \cdot B_{m} \cdot k_{c} \cdot S_{c}}{2^{\frac{1}{2}}}
$$

Where

$\omega$ - The angular frequency of the current's winding;

$\Phi_{m}$-Magnetic flux, coupled with a heating coil.

$B_{m}$ - The induction of the core;

$k_{c}-$ A coefficient, that takes into account the insulation between the plates. of the core

Replacing $\mathrm{E}_{\mathrm{k}}$ by (13) in (10) and using (6), (9) and (12) we can get

$$
R_{k}=\left(1,4 \cdot 2 \cdot \frac{C \cdot \gamma \cdot \rho_{A}}{k_{c} \cdot B_{m}^{2}}(2 \pi+6)\left(\theta_{2}-\theta_{1}\right) \frac{v}{\omega^{2}}\right)^{\frac{1}{3}}=K_{R}\left(\frac{v}{\omega^{2}}\right)^{\frac{1}{3}}
$$

Taking that into consideration

$$
\begin{aligned}
& C=910 \mathrm{~J} / \mathrm{kg} .{ }^{0} \mathrm{C} ; \\
& \gamma=2700 \mathrm{~kg} / \mathrm{m}^{3} ; \\
& \mathrm{P}_{\mathrm{a}}=\rho_{A}=2,62.10^{-8} \mathrm{OM} . \mathrm{M} ; \\
& k_{c}=0.95 .
\end{aligned}
$$

When limiting $B_{m}$ in the value of the inductions' saturation, which for the cold rolled steel reaches up to 1.5 $\mathrm{Tl}$, equation (14) turns into

$$
R_{k}=6,36\left(\frac{v}{\omega^{2}}\right)^{\frac{1}{3}}
$$

This way, by the given performance of the heat treatment process, and the selected frequency of the inductor coil current by equation (15) we calculate the radius of the deflecting roller $R_{k}$. Further, with a known $R_{k}$ by (7) we calculate the cross sectional dimensions of both terminals of the core. Length of the rod $1_{0}$ (fig.1) can be found through the condition of placing the winding.

In a steady thermal state of the inductors' winding, power loss $\Delta \mathrm{P}$ completely passes to the surrounding environment through its external surface, which is expressed by the famous formula of 


$$
\Delta P=k_{T} S_{\sigma} \cdot \tau
$$

Where

$k_{T}-$ Heat transfer coefficient;

$S_{\sigma}$ - The surface area, through which heat is transferred to a cooling medium;

$\tau$ - The difference of temperature between the surface and the cooling media.

Raising the temperature of the surface of the winding over the temperature of the cooling medium $\tau$ should not be higher than the permissible value, set by the standards. During the preliminary calculations, it is usually taken, that heat transfer only takes place via the lateral surface of the outer coil. From (picture.1) the area of the lateral outer surface of the coil is

$S_{\sigma}=\left(8 \cdot k_{1} \cdot R_{k}+2 \pi \cdot b_{0}\right) \cdot l_{0}$

Where $l_{0}$ - the axial length of the winding, equal to the length of the rods' core.

Heat flux, equals $k_{T} \tau$, to the outer side surface of the coil dry-type transformers doesn't exceed $600 B_{T} / \mathrm{m}^{3}$, raising the temperature of shouldn't get over $60^{\circ} \mathrm{C}$.

In order to find the size of the axial section of the winding $b_{0}$ and $1_{0} l_{0}$ we can use the expression of the volume occupied by the winding (fig.1)

$V_{0}=\left(8 \cdot k_{1} \cdot R_{k} \cdot b_{0}+\pi \cdot b_{0}^{2}\right) \cdot l_{0}$

Looking into the surface of the outer side of the winding $S_{\sigma}$, as the predetermined value, the last expression given in consideration of (17) takes the form of

$V_{0}=S_{\sigma} \cdot b_{0}-\pi b_{0}^{2} l_{0}$

The maximum volume, occupied by the coil, when limiting the surface area of the cooling guarantees a

minimum current density in the winding wires. Expression (19) reaches its maximum when $b_{0 m}=S_{\sigma} / 2 \pi l_{0}$, from which the area, taken by the winding of the core

$S_{0}=b_{0 m} l_{0}=\frac{S_{\sigma}}{2 \pi}$

The calculation of $l_{0}$ by (20), can be done under the condition, that the ratio $\beta=\frac{l_{0}}{b_{0}}$ is set.

At $b_{0 m}=\frac{l_{0}}{\sigma}$ from the expression (20) it turns out that

$l_{0}=\left(\frac{S_{\sigma} \cdot \beta}{2 \pi}\right)^{\frac{1}{2}}$

Power loss in the winding, taking (5) into consideration takes the form of

$\Delta P=I^{2} \cdot \rho_{M}\left[1+\alpha\left(\tau+\theta_{0}\right)\right] \cdot \frac{l_{c p}}{k_{3} S_{0}}$

Where

$\rho_{M}-$ The resistivity of copper ;

$\theta_{0}-$ The temperature of the cooling medium;

$k_{3}$-The fill factor of the winding;

$I$ - the referred value of flowing current winding;

Using the expression (8), (20), and (21), the latter can lead to the following form

$$
\Delta P=I^{2} \cdot \rho_{M}\left[1+\alpha\left(\tau+\theta_{0}\right)\right] \cdot \frac{8 k_{1} R_{k} \cdot \beta+\pi l_{0}}{k_{3} S_{0}}
$$

The equation of the M.D.S equilibrium of the inductor's winding and the heating coil, if we omit its losses in the rods and yoke core it has a simple form and can be used to calculate the present coil current. 


$$
F_{m}=\frac{\omega \cdot \phi_{m}}{R_{3}}=\frac{\omega \cdot B_{m} \cdot k_{c} S_{c}}{R_{3}}
$$

By replacing, $S_{c}, R_{3}$ and $R_{k}$ by (9), (12) and (15) the last expression becomes

$$
F_{m}=0.348 \frac{B_{m} \cdot q}{\rho_{A}} \cdot(\omega \cdot v)^{\frac{1}{3}}
$$

The sequential substitution in (21) $S_{\sigma}$ by (16) and $\Delta P$ by (23) gives and expression to calculate $1_{0}$ an equation of the fourth degree, for which the analytic solution is impossible.

The more suitable method for getting solutions is modeling. The schemes of the model, built in the software environment of MATLAB 6.5, shown in fig.2.

Research on the model has shown, that $\beta$ should be chosen of a value not less than 2.5 , smaller values result in an increase in losses, due to the increasing length of the average coil. The cross sectional area of the yoke and the rod are usually equal.

The length of the yoke is chosen by the condition and the amenities available for filling the wire in the inductor and the thermal insulation of the surface of the winding from the hot wire.

The dimensions of the winding, selected for the proposed technique, provide a complete flow at the level of acceptable values for a dry-type transformer.

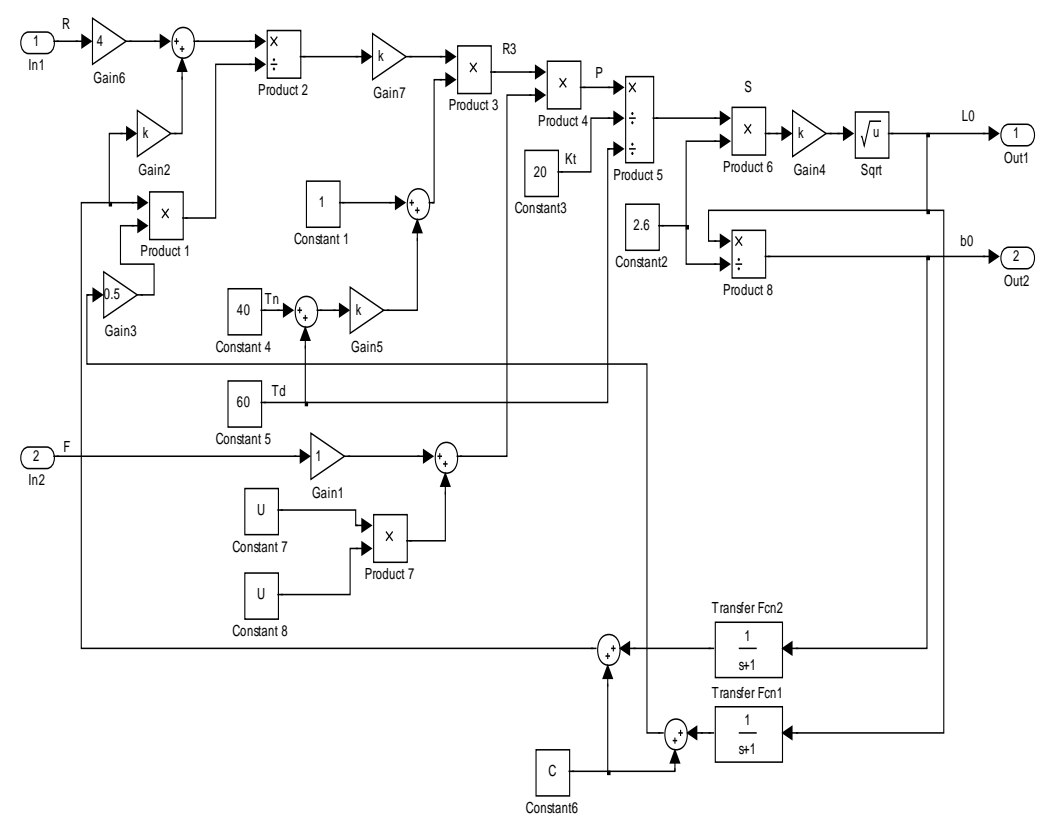

Fig.2. Model of $l_{0}$ and $b_{0}$ calculations.

Notes:

Main relationship:

To find the size of the axial section of the winding $b_{0}$ and $l_{0}$ we can use the expression of the volume occupied by the winding (picture.1)

$V_{0}=\left(8 \cdot k_{1} \cdot R_{k} \cdot b_{0}+\pi \cdot b_{0}^{2}\right) \cdot l_{0}$

\section{Results, Discussion, and Conclusion}

Fig.3 shows the relation between the size of the conductor and the axial section of the windings. It is found that the winding size increases as the axial section of the wire increases, the relation is nearly linear, but at axial section of 12-14 the winding size decreases but after that it is continued increasing. In general winding size is proportional to axial section. 


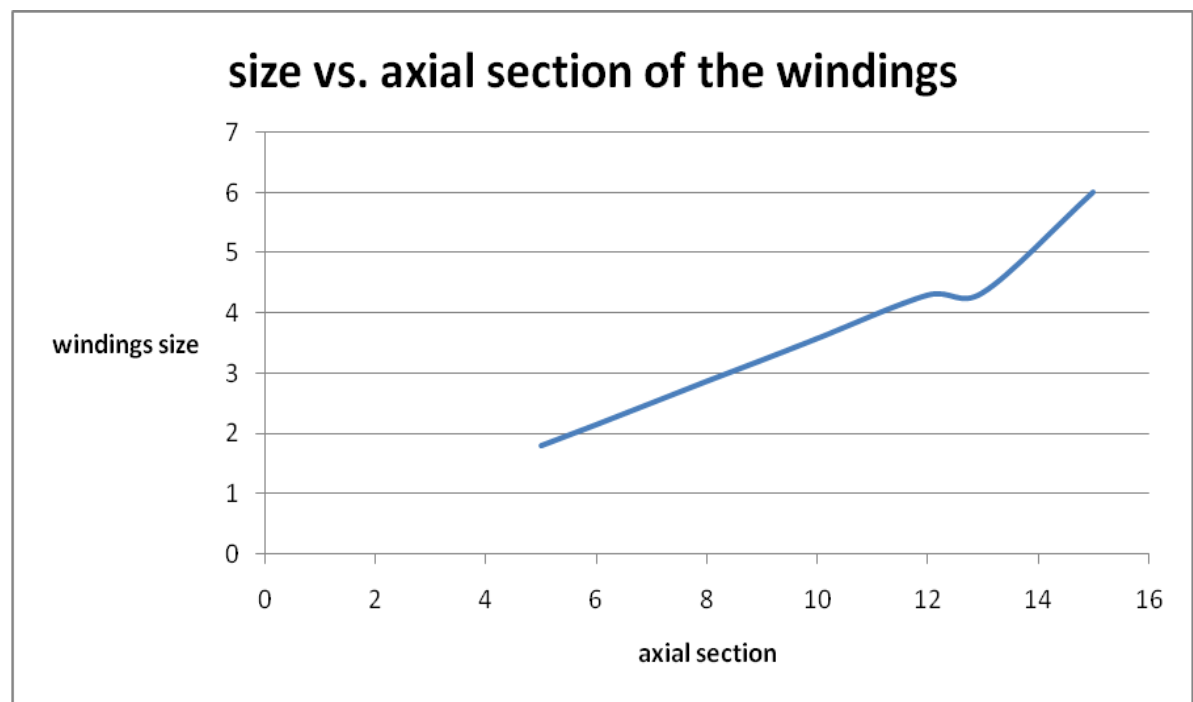

Fig.3 Size of the windings vs. axial section.

\section{CONCLUSIONS}

1- Research on the model has shown, that $\beta$ should be chosen of a value not less than 2.5 , smaller values result in an increase in losses, due to the increasing length of the average coil. The cross sectional area of the yoke and the rod are usually equal.

2- The length of the yoke is chosen by the condition and the amenities available for filling the wire in the inductor and the thermal insulation of the surface of the winding from the hot wire.

3- The dimensions of the winding, selected for the proposed technique, provide a complete flow at the level of acceptable values for a dry-type transformer.

\section{REFERENCES:}

[1] Theory of heating by induction, 2001 ASM Intrnational. Practical induction heat treating (\# 06098G). WWW.asminternational.org.

[2] Xi Nan; Sullivan, C. R. 2005, An equivalent complex permeability model for litz- wire windings. Industry application conference 3, p: 2229-2235 vol.3, IEEE.

[3] Skilling, Hough H. (1951), Electric transmition lines, Mc Graw-Hill.

[4] Hayt, William (1981), engineering electromagnetic (4 ${ }^{\text {th }}$ ed.), Mc Graw-Hill, isbn 0-07-027395-2.

[5] Aluminium electrical conductor hand book, $3^{\text {rd }}$ ed. 1989. The aluminium association $19^{\text {th }}$ street, N. W., Washington, D. C.2006.

[6] Robert L. Doney, George B. Vunni, and John H. Niederhaus, 2010,

a. Experiments and Simulations of Exploding Aluminum Wires: Validation of ALEGRA-MHD, army research lab.

[7] 7. Rosenthal, S.E.; Desjarlais, M.P.; Cochrane, K.E. Equation of State and

[8] Electron Tranport Effects in Exploding Wire Evolution; SAND2001-1896C,

[9] 2001.

[10] Degnan, J.H.; Taccetti J.M. Implosion of solid liner for compression of field reversed configuration. IEEE Trans. Plasma Sci. 2001, 29, pp. 93-98.

[11] Desjarlais, M.P.; Rosenthal, S.E.; Douglas, M.R.; Cochrane, K.; Spielman, R.B.; Mehlhorn, T.A. Study of Conductivity and EOS Variations in Exploding Wire Simulations. GP1.066 Poster Session IV, 42nd Annual Meeting of the APS Division of Plasma Physics, Quebec City, Canada, October 23-27, 2000.

[12] Mehlhorn, T.A.; Stoltz, P.H.; Haill, T.A.; Desjarlais, M.P.; Douglas, M.R. Verification and Validation of ALEGRA-MHD on exploding wire data. GP1.065 Poster Session IV, 42nd Annual Meeting of the APS Division of Plasma Physics, Quebec City, Canada, October 23-27, 2000.

[13] Sarkisov, G.S.; Rosenthal, S.E.; Cochrane, K.R.; Struve, K.W.; Deeney, C.; McDaniel, D.H. Nanosecond electrical explosion of thin aluminum wires in a vacuum: Experimental and computational investigations. Phys. Rev. E. 2005, 71, 046404. 